\title{
Identification of genes regulating ovary differentiation after pollination in hazel by comparative transcriptome analysis
}

\author{
Yunqing Cheng ${ }^{1}$, Yuchu Zhang ${ }^{1}$, Chunming Liu', Pengfei $\mathrm{Ai}^{2}$ and Jianfeng Liü ${ }^{1 *}$
}

\begin{abstract}
Background: Hazel (Corylus spp.) exhibits ovary differentiation and development that is initiated from the ovary primordium after pollination, conferring the plant with a unique delayed fertilization. Failure of development of the ovary and ovule after pollination can lead to ovary abortion and blank fruit formation, respectively, with consequent yield loss. However, the genes involved in ovary and ovule differentiation and development are largely unknown.

Results: In unpollinated pistillate inflorescences (stage F), the stigma shows an extension growth pattern. After pollination, a rudimentary ovary begins to form (stage S), followed by ovule differentiation (stage $\mathrm{T}$ ) and growth (stage FO). Total RNA was obtained from pistillate inflorescences or young ovaries at stage F, S, T and FO, and sequencing was carried out on a HiSeq 4000 system. De novo assembly of sequencing data yielded $62.58 \mathrm{~Gb}$ of nucleotides and 90,726 unigenes; 5524, 3468, and 8714 differentially expressed transcripts were identified in F-vs-S, S-vs-T, and T-vs-FO paired comparisons, respectively. An analysis of F-vs-S, S-vs-T, and T-vs-FO paired comparisons based on annotations in the Kyoto Encyclopedia of Genes and Genomes revealed six pathways that were significantly enriched during ovary differentiation, including ko04075 (Plant hormone signal transduction). Auxin level increased after pollination, and an immunohistochemical analysis indicated that auxin was enriched at the growth center of pistillate inflorescences and young ovaries. These results indicate that genes related to auxin biosynthesis, transport, signaling, the floral quartet model, and flower development may regulate ovary and ovule differentiation and development in hazel.
\end{abstract}

Conclusions: Our findings provide insight into the molecular mechanisms of ovary differentiation and development after pollination in this economically valuable plant.

Keywords: Hazel, Ovary, Ovule, Transcriptome

\section{Background}

Hazel (Corylus spp.) is the most economically important plant of the Betulaceae family. Turkey and Italy produce more than $80 \%$ of the world's hazelnut crop. Other important hazelnut producers are the U.S. (3.8\%), Azerbaijan (3.3\%), Spain (2.6\%), Iran (1.9\%), Georgia (1.9\%), and China (1.7\%) [1]. In the last decade, the area devoted to the culture of hybrid hazel $(C$. heterophylla $\times C$. avellana) has increased dramatically in China and is currently more than $50,000 \mathrm{hm}^{2}$ with a concomitant increase in fruit yield, setting the stage for China to become a major global

\footnotetext{
*Correspondence: jianfengliu1976@163.com

${ }^{1}$ Jilin Provincial Key Laboratory of Plant Resource Science and Green

Production, Jilin Normal University, Siping 136000, Jilin Province, China

Full list of author information is available at the end of the article
}

producer of hazelnuts. Northeast China is the largest production area, with the hazelnut industry significantly impacting regional economic development.

In angiosperms, stigma growth, pollination, fertilization, and subsequent ovary and ovule development in pistillate inflorescences are the requirements for fruit formation, which determines plant yield. The mature gynoecium has four main components: stigma, style, ovary, and gynophore [2]. In general, the mature gynoecium at anthesis is ready for fertilization [3]. Compared to Arabidopsis, hazel has distinct pistillate inflorescences and a different mode of fruit development: at anthesis of pistillate inflorescences, the gynoecium is still immature due to the absence of a complete ovary and ovule, and the stigma and style form a complex [4]; at the bottom of this

(c) The Author(s). 2018 Open Access This article is distributed under the terms of the Creative Commons Attribution 4.0 International License (http://creativecommons.org/licenses/by/4.0/), which permits unrestricted use, distribution, and 
complex are a few layers of ovary primordium cells $[5,6]$. After pollination, pollen tubes reach to the bottom of the style, which is also known as the pollen tube cavity. The early ovary and ovule primordium then begin to differentiate. After mature embryo sacs are formed, pollen tubes regrow and enter the ovary, and complete fertilization about 2 months after pollination [6]. Pollen tube growth signaling has an important impact on ovary and ovule differentiation in hazel. Ovary and ovule abortion are common during pistillate inflorescence and fruit development [7, 8]. Ovary abortion is partly caused by the absence of a mature embryo sac, and determines the number of fruit per cluster [5]. A complete embryo structure can be observed in an abortive ovule, which is closely associated with blank fruit formation [8]. Most of the genes that regulate ovary and ovule development in hazel are not known. Given the unique pistillate inflorescence and fruit development characteristics of this plant, identifying these genes will provide insights into the mechanism of ovary development and yield loss caused by frequent ovary and ovule abortion during the fruit development stage.

In the present study, we analyzed gene expression in pistillate inflorescences and young fruits during four developmental stages by Illumina HiSeq 4000 analysis. We identified differentially expressed genes (DEGs) that may be involved in the regulation of ovary and ovule formation in hazel. Auxin was detected in pistillate inflorescences and young fruits by immunohistochemistry and HPLCMS/MS (high-performance liquid chromatographytandem mass spectrometry) in order to clarify its function in ovary development. Comparison of gene expression patterns at the four developmental stages of the ovary provides important insights into the molecular mechanisms of ovary differentiation and growth after pollination in hazelnut.

\section{Methods}

\section{Plant material and treatment}

The field experiment was conducted at a hazel orchard near Siping, Jilin Province, China. The experimental site is located in the North Temperate Zone and has a typical continental monsoon climate. Local hazel blooming and harvest dates are approximately April 20 and August 25, respectively.

In 2015, sixty 12-year-old hybrid hazel trees $(C$. heterophylla $\times$ C. avellana) cultivar 'Dawei', from Forest Research Institute of Liaoning Province, Dalian, China, were selected as study materials. On March 20 of that year, staminate shoots of the 'PingOu 21', 'Yuzhui', and 'Liaozhen 7' cultivars from Forest Research Institute of Liaoning Province were sampled for artificial pollination only and cultured in a light incubator; pollen was collected and the pollen germination ratio was determined as previously described [5]. After mixing in equal quantities, the pollen was stored at $4{ }^{\circ} \mathrm{C}$ until used for artificial pollination. On April 8, about 3000 pistillate inflorescences of 'Dawei' were randomly bagged and tagged. On April 20 (blooming date), about 1500 pistillate inflorescences were collected as unpollinated samples (stage F). Artificial pollination was carried out on the same day, and about 1000 pistillate inflorescences or young fruit clusters were sampled on May 10 when a rudimentary ovary began to develop (stage S). On May 30 , about 120 pistillate inflorescences were sampled (early ovule formation, stage T); and on June 20, about 60 fruit clusters were sampled (achievement of fertilization and ovule growth, stage FO). After transport to the laboratory, the exterior bracts of pistillate inflorescences or fruit clusters were manually removed, and the stigma with the ovary primordium or ovary was isolated and collected, with three biological replicates prepared for each developmental stage. The samples were stored in liquid nitrogen until use. The voucher specimens of these materials have been publicly deposited in Shenyang Agriculture University, Shenyang, China. All field experiments were performed in accordance with the Convention on the Trade in Endangered Species of Wild Fauna and Flora.

\section{RNA extraction, library construction, and sequencing}

In order to investigate changes in gene expression during the four developmental stages, 12 digital gene expression (DGE) profiling libraries were constructed: DWN-F-A (DWN: hybrid hazelnut cultivar 'Dawei'), DWN-F-B, DWN-F-C, DWN-S-A, DWN-S-B, DWN-SC, DWN-T-A, DWN-T-B, DWN-T-C, DWN-FO-A, DWN-FO-B, and DWN-FO-C. For the library names, 'F,' 'S', 'T', and 'FO' indicate first, second, third, and fourth developmental stages and 'A', 'B', and ' $\mathrm{C}$ ' indicate the three biological replicates. Total RNA was extracted from pistillate inflorescences or young ovaries using the RNA Easy spin Isolation System (Aidlab Biotech, Beijing, China). RNA pretreatment before sequencing was performed as previously described [9], and sequencing was carried out on a HiSeq 4000 system (Illumina, San Diego, CA, USA). Raw transcriptome data were deposited in the Sequence Read Archive (https://www.ncbi. nlm.nih.gov/SRA).

\section{Data analysis and mapping of DGE tags}

After removal of reads with adaptors, reads with more than 5\% unknown bases $(\mathrm{N})$, and low quality reads (defined as reads in which the proportion of bases with a quality score $<10$ was greater than $20 \%$ ), the remaining clean reads were stored in FASTQ format. Trinity (version: v2.0.6) [10] was used to perform de novo assembly with clean reads from all 12 libraries with min contig_length set to 150 and min_kmer_cov set to 3 and 
all other parameters set to default. Tgicl (version: v2.0.6) [11] was used to cluster transcripts to Unigenes with repeat_stringency, minmatch, and minscore set to $0.95,35$, and 35, respectively, and all other parameters set to default. We used Blast [12] to align unigenes to NT, NR, COG, KEGG and SwissProt to obtain annotations, Blast2GO (Version: v2.5.0; parameters: default) [13] with NR annotation to obtain GO annotations, and InterProScan5 [14] to obtain InterPro annotations. Clean reads were mapped to unigenes using Bowtie2 (version: v2.1.0) [15] with options "q; phred64; sensitive; dpad, 0; gbar, 99999999; mp, 1, 1; np, 1; score-min L, 0, -0.1; I, 1; X, 1000; no-mixed; no-discordant; p, 1; k, 200", and then, gene expression levels were calculated with RSEM [16]; all parameters were set to default. For different sample libraries (F-vs-S, S-vs-T, and T-vs-FO, in which the former was used as the control and the latter as the experimental group in each paired comparison), three pairs of DGE profiles were compared in order to determine changes in gene expression during ovary differentiation and ovule growth in hazel. Based on the unigene expression results, DEGs were identified with EBseq [17] by setting the threshold of fold change as $\geq 2.00$ and posterior probability of equivalent expression (PPEE) as $\leq 0.05$. KEGG pathway enrichment analysis of DGE data was carried by a BLAST search of the KEGG database (http://www.kegg.jp/kegg/). Q $\leq 0.05$ was used as the threshold for significant enrichment of DEG KEGG pathways. Hierarchical clustering analysis of DEGs was carried out using Multi Experiment Viewer (http://mev. tm4.org/\#/welcome).

\section{Auxin localization and quantification}

Fresh hazel ovary or stigma samples from four different developmental stages were fixed in $4 \%$ paraformaldehyde for $16 \mathrm{~h}$ at $4{ }^{\circ} \mathrm{C}$, then washed with phosphate-buffered saline (PBS) and incubated in $2.0 \mathrm{M} \mathrm{NaOH}$ for $30-40 \mathrm{~min}$ at $65{ }^{\circ} \mathrm{C}$. The softened samples were incubated in $0.1 \mathrm{M}$ acetic acid for $30 \mathrm{~s}$, rinsed three times with distilled water, boiled in $0.01 \mathrm{M}$ sodium citrate buffer ( $\mathrm{pH}$ 6.0) for antigen retrieval, and blocked in PBS with Tween and $1 \%$ bovine serum albumin. Anti-auxin antibody $\left(1 \mathrm{mg} / \mathrm{ml}^{-1}\right.$ ) (Sigma-Aldrich, St. Louis, MO, USA) and fluorescein isothiocyanate- conjugated goat anti-mouse IgG $(\mathrm{H}+\mathrm{L})$ (Beyotime Institute of Biotechnology, Nanjing, China) were used as primary and secondary antibodies, respectively. Samples were mounted with anti-fade mounting medium (Beyotime Institute of Biotechnology) and cover slips, and imaged under a fluorescence microscope (Olympus, Tokyo, Japan) at an excitation wavelength of $494 \mathrm{~nm}$.

The levels of auxin were determined by Zoonbio Biotechnology Co., Ltd. (Nanjing, China) using HPLCMS/MS [18]. Auxin extraction and purification was performed as previously described using $0.5 \mathrm{~g}$ frozen samples. Then, the purified product was subjected to HPLC-MS/MS analysis. HPLC (Agilent 1260) analysis was carried out using a ZORBAX SB-C18 (Agilent Technologies) column $(2.1 \mathrm{~mm} \times 150 \mathrm{~mm} ; 3.5 \mu \mathrm{m})$. The mobile phase A solvent consisted of water $/ 0.1 \%$ methanoic acid, and the mobile phase $B$ solvent consisted of ultrapure methanol. The injection volume was $10.0 \mu \mathrm{L}$. Parameters for MS analysis were set as follows: spray voltage, $+4500 \mathrm{~V},-3000 \mathrm{v}$; pressure of the air curtain, nebulizer, and aux gas were 15,65 , and $70 \mathrm{psi}$, respectively; atomizing temperature, $350{ }^{\circ} \mathrm{C}$. Measurements were obtained for three biological replicates of each sample. Data were analyzed using SAS v.8.01 (SAS Institute, Cary, NC, USA) and significant differences between groups were evaluated with the least significant difference (LSD) test at a significance level of $5 \%$.

\section{Quantitative real-time (qRT)-PCR analysis}

The DGE results obtained by Illumina HiSeq 4000 sequencing were validated by qRT-PCR as previously described, using the same RNA samples [7]. Relative expression levels of selected genes were calculated with the $2^{-\Delta \Delta C t}$ method [7]; primers used for qRT-PCR analysis are listed in Additional file 1: Table S1.

\section{Results}

\section{Illumina sequencing and sequence assembly}

To evaluate global changes in gene expression during ovary differentiation and ovule growth in hazel, 12 DGE libraries were sequenced with the Illumina HiSeq 4000 platform, generating approximately 52 million bases of raw reads for each library. The low-quality reads were filtered, yielding 625.79 million bases of clean reads. For all 12 libraries, de novo assembly of clean reads generated 62.58 Gb of nucleotides and 90,726 unigenes with a mean length of 1054 bp (Additional file 2: Table S2). All raw transcriptome data were deposited in the sequence read archive (accession no. SRS1980648). A query of unigenes against seven public nucleotide and protein databases yielded 67,946 (74.89\%) annotated unigenes (Table 1).

\section{Global gene expression at four different developmental stages of ovaries}

In total, 56,605, 63,281, 63,450, and 60,410 unigenes were recovered at stages F, S, T, and FO of ovary development, respectively (Fig. 1). The number of expressed genes was higher at stages $\mathrm{S}$ and $\mathrm{T}$ than at stages $\mathrm{F}$ and $\mathrm{FO}$, indicating that more genes are needed to coordinate the initiation of the rudimentary ovary after pollination and ovule differentiation. Constitutive expression of 45,575 genes was found in all four developmental stages; 2013, 1940, 2198, and 1680 were expressed only at stages $F, S, T$, and FO, respectively. Genes with stage- 
Table 1 Summary of functional annotations

\begin{tabular}{llllllllll}
\hline Values & Total & $\begin{array}{l}\mathrm{Nr} \\
\text { Annotated }\end{array}$ & $\begin{array}{l}\text { Nt } \\
\text { Annotated }\end{array}$ & $\begin{array}{l}\text { SwissProt } \\
\text { Annotated }\end{array}$ & $\begin{array}{l}\text { KEGG } \\
\text { Annotated }\end{array}$ & $\begin{array}{l}\text { COG } \\
\text { Annotated }\end{array}$ & $\begin{array}{l}\text { InterPro } \\
\text { Annotated }\end{array}$ & $\begin{array}{l}\text { GO } \\
\text { Annotated }\end{array}$ & Overall \\
\hline Number & 90,726 & 60,659 & 60,865 & 42,927 & 37,636 & 26,110 & 47,356 & 11,596 & 67,946 \\
Percentage & $100 \%$ & $66.86 \%$ & $67.09 \%$ & $47.31 \%$ & $41.48 \%$ & $28.78 \%$ & $52.20 \%$ & $12.78 \%$ & $74.89 \%$ \\
\hline
\end{tabular}

NT, nucleotide sequence database, website: ftp://ftp.ncbi.nlm.nih.gov/blast/db; NR, non-redundant protein sequence database, website: ftp://ftp.ncbi.nlm.nih.gov/ blast/db; GO, Gene Ontology, website: http://geneontology.org; COG: Cluster of Orthologous Groups of proteins, website: http://www.ncbi.nlm.nih.gov/COG; KEGG: Kyoto Encyclopedia of Genes and Genomes, website: http://www.genome.jp/kegg; SwissProt: UniProt Knowledgebase, website: http://ftp.ebi.ac.uk/pub/databases/ swissprot; InterPro: InterPro database, website: http://www.ebi.ac.uk/interpro

specific expression are presumed to coordinate stigma development, rudimentary ovary development, ovule differentiation, and ovule growth. The ovule differentiation and ovule growth stages had the largest and smallest number of stage-specific genes, respectively.

\section{DEGs in paired comparisons of four different ovary development stages}

A fold change threshold of $\geq 2.00$ and a PPEE threshold of $\leq 0.05$ were used to identify DEGs in the three paired comparisons (Fig. 2; Table 2). Similar numbers of expressed genes were found in the paired comparisons of F-vs-S $(68,046)$, S-vs-T $(68,794)$, and T-vs-FO $(67,926)$ (Table 2). The number of DEGs in the F-vs-S paired comparisons was 5524 (2522 downregulated and 3002 upregulated; Additional file 3: Table S3); 3468 DEGs were found in the S-vs-T paired comparisons (1970 downregulated and 1498 upregulated; Additional file 4: Table S4); and 8714 DEGs were found in the T-vs-FO paired comparisons (4427 downregulated and 4287 upregulated; Additional file 5: Table S5). Thus, the highest number of DEGs was found in the T-vs-FO paired comparisons. The rudimentary ovary begins to form at stage $\mathrm{S}$ after pollination, and ovary and ovule sizes increase

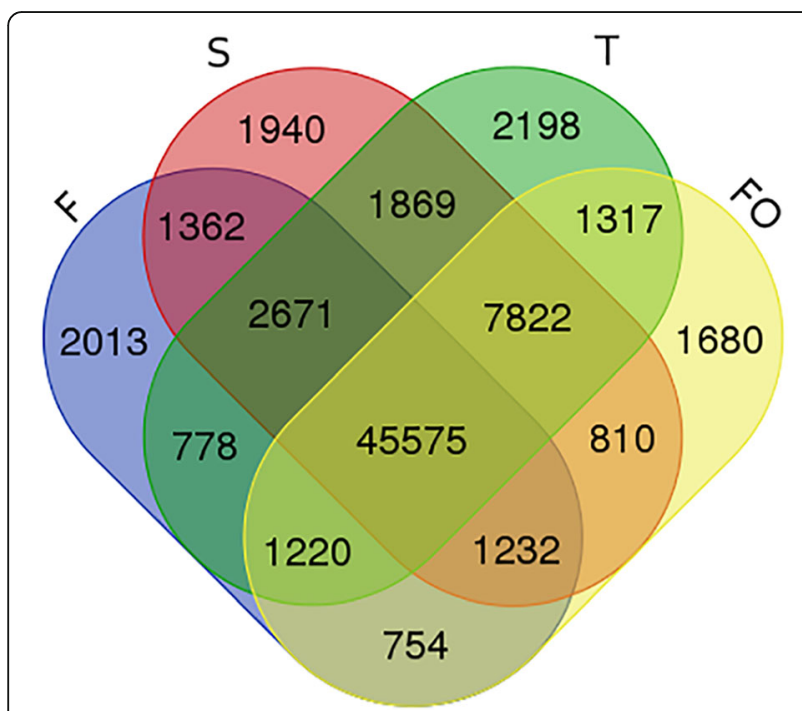

Fig. 1 Venn diagram showing the overlaps of gene expression between the four different developmental stages in hybrid hazelnut from stage $\mathrm{T}$ to $\mathrm{FO}$. Our results indicate that many genes are activated upon pollination at stage $\mathrm{S}$ compared to stage $F$, as evidenced by the large number of upregulated DEGs, in order to meet the demands for material and energy during ovule growth at stage FO.

\section{Analysis of DEG clustering and pathway enrichment}

Hierarchical clustering analysis of DEGs in F-vs-S $(5,524)$, S-vs-T $(3,468)$, and T-vs-FO (8714) paired comparisons identified only 164 transcripts common to all three comparisons (Fig. 3); this is presumably insufficient for regulating the conversion of several layers of ovary primordium at stage $\mathrm{S}$ to an ovary with developing ovules at stage FO, and implies that stage-specific transcripts are also involved.

A KEGG pathway classification and functional enrichment analysis were carried out for DEGs identified in the F-vs-S, S-vs-T, and T-vs-FO paired comparisons to determine the pathways involved in the regulation of ovary differentiation. In total, 39 pathways with a $\mathrm{Q}$ value $<0.05$ were identified: 25 pathways were significantly enriched in F-vs-S, 25 in S-vs-T, and 13 in T-vsFO (Table 3; Additional files 6, 7 and 8: Tables S6, S7 and S8). Six significantly enriched pathways were common to the three paired comparisons (Fig. 4), including ko00940 (Phenylpropanoid biosynthesis), ko01110 (Biosynthesis of secondary metabolites), ko00941 (Flavonoid biosynthesis), ko00945 (Stilbenoid, diarylheptanoid, and gingerol biosynthesis), ko04075 (Plant hormone signal transduction), and ko00073 (Cutin, suberine, and wax biosynthesis), which are involved in the regulation of metabolism and signal transduction. These results indicate that DEGs involved in biosynthesis, metabolism, and signal transduction are important for the regulation of ovary differentiation and development in hazel.

\section{DEGs in auxin biosynthesis, transport, and signal transduction pathway}

We identified three unigenes encoding YUC (Indole-3pyruvate monooxygenase) with relatively high fragments per kilobase of transcript per million mapped read (FPKM) values and significantly altered expression. In the F-vs-S paired comparisons, the three unigenes encoding YUC (CL11083.Contig2_All, Unigene15073_All, and CL7285.Contig3_All) were upregulated 1.62, 1.63, 

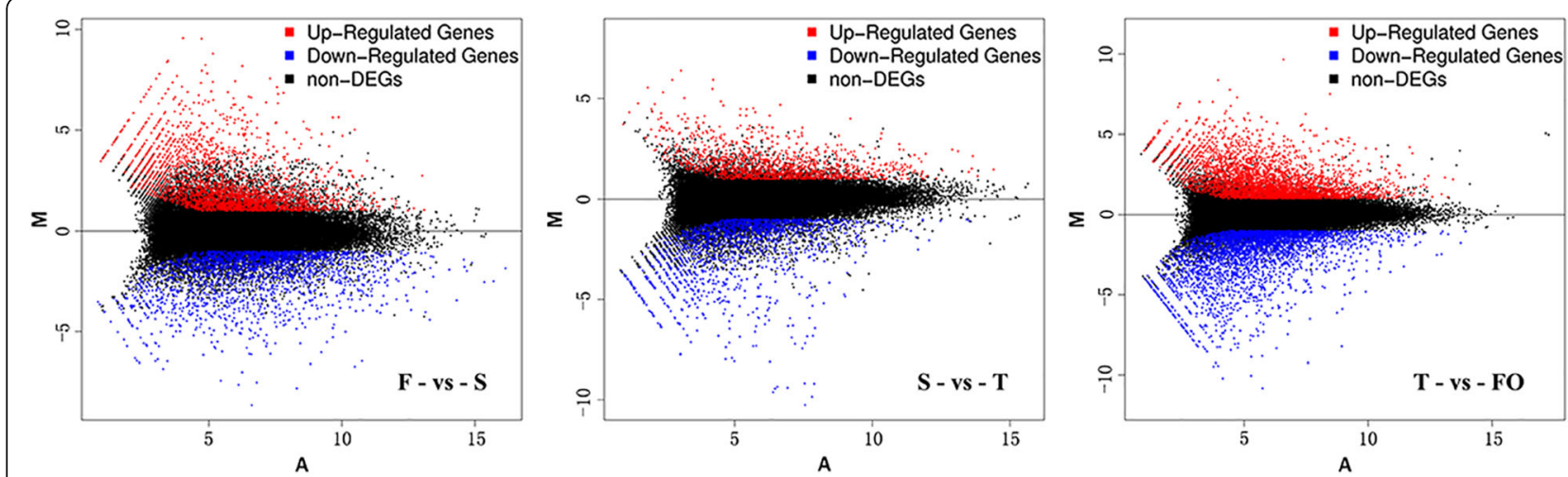

Fig. 2 MA plot of DEGs. The MA-plot is a plot of the distribution of the red/green intensity ratio ('M') plotted by the average intensity ('A'). $M$ is the binary logarithm of the intensity ratio (or difference between log intensities) and $\mathrm{A}$ is the average log intensity for a dot in the plot. $\mathrm{X}$ axis represents value $A$ (log2 transformed mean expression level), and $Y$ axis represents value $M$ (log2 transformed fold change). Red, blue and black points represent up-, down- and non-regulated DEGs respectively. "F" was the control and "S" was experimental group in "F-vs-S" paired comparison

and 1.32-fold, respectively (Additional file 9: Table S9). The FPKM values of Unigene15073_All were the highest among the three DEGs $(5.47,18.84,22.39$, and 19.46 at stages F, S, T, and FO, respectively) (Additional file 10: Table S10), indicating high $Y U C$ expression levels after the first stage of development. Consistent with this observation, a DEG encoding the auxin efflux carrier family protein PIN-FORMED (PIN) (CL2654.Contig1_All) was upregulated 1.47 -fold in the F-vs-S paired comparisons, with FPKM values at stages F, S, T, and FO of 8.73, 26.71, 16.78, and 20.25, respectively (Additional files 9 and 10: Tables S9 and S10). Thus, YUC and PIN were highly expressed from stage $\mathrm{S}$.

Our transcriptome data revealed dozens of DEGs in the auxin signal transduction pathway (Additional file 9: Table S9). Among the five DEGs encoding auxin influx carrier protein 1 (AUX1), the maximum FPKM value of Unigene23267_All at the four developmental stages was much higher than those of the other four DEGs (Additional file 10: Table S10). FPKM was maximal at stage $F$, and then decreased and remained at a relatively low level at subsequent developmental stages. Only one DEG encoding TRANSPORT INHIBITOR RESPONSE 1 (TIR1) (CL9570.Contig1_All) was identified, and its FPKM values were 9.57, 8.93, 15.30, and 6.09 at stages F, S, T, and FO, respectively (Additional file 10: Table S10),

Table 2 Gene expression levels across different sample libraries

\begin{tabular}{llll}
\hline Differently expressed genes & F-vs-S & S-vs-T & T-vs-FO \\
\hline Up-regulated & 3002 & 1498 & 4287 \\
Downregulated & 2522 & 1970 & 4427 \\
Total DEGs & 5524 & 3468 & 8714 \\
Not DEGs & 62,522 & 65,326 & 59,212 \\
Total expressed genes & 68,046 & 68,794 & 67,926 \\
\hline
\end{tabular}

indicating that pollination at stage $\mathrm{S}$ had no significant impact on expression.

AUX/IAA (auxin-responsive protein AUX/IAA) behaves as a negative regulator in auxin signaling. All three DEGs (CL1889.Contig2_All, Unigene10494_All, and CL1889.Contig1_All) encoding $A U X / I A A$ were markedly downregulated in F-vs-S paired comparisons (Additional file 9: Table S9). The FMKP value of each DEG decreased and remained at a low level at later developmental stages (Additional file 10: Table S10). These results indicate that AUX/IAA may block auxin

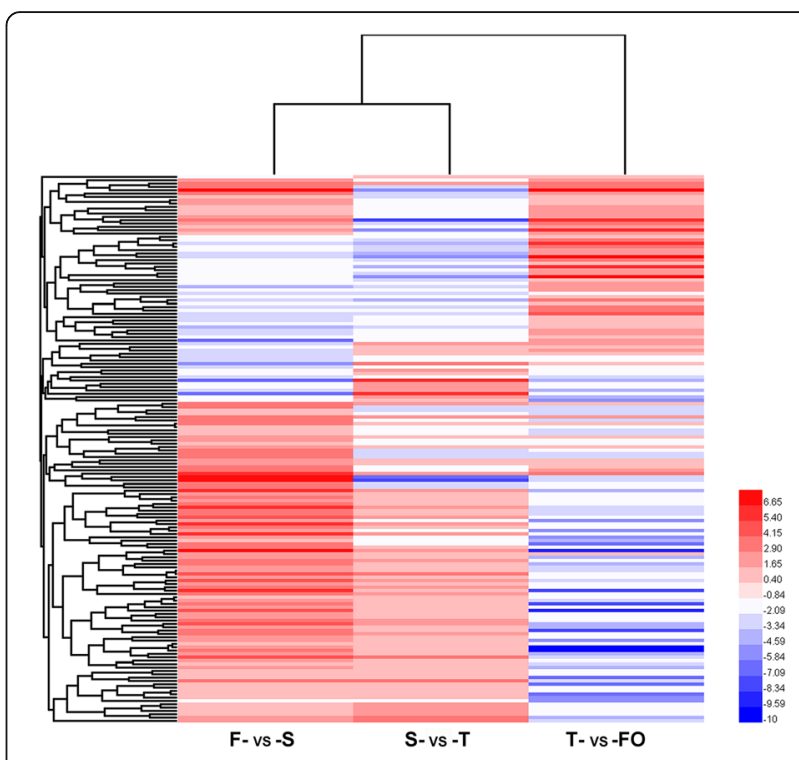

Fig. 3 Hierarchical clustering (HCE) analysis of differentially expressed transcripts in F-vs-S, S-vs-T, and T-vs-FO paired comparisons. "F" was the control and "S" was experimental group in "F-vs-S" paired comparison. Each line refers to data from one gene. The color bar represents the $\log _{10}$ RPKM and ranges from blue (low expression) to red (high expression) 
Table 3 Common significant enriched pathways in F-vs-S, S-vs-T and T-vs-FO paired comparisons

\begin{tabular}{llllllll}
\hline KO ID & Pathway name & & DEGs & & Q value \\
& & F-vs-S & S-vs-T & T-vs-FO & F-vs-S & S-vs-T & T-vs-FO \\
\hline ko00940 & Phenylpropanoid biosynthesis & $110(3.3 \%)$ & $109(5.4 \%)$ & $148(2.84 \%)$ & $7.20 \mathrm{E}-09$ & $3.09 \mathrm{E}-23$ & $1.01 \mathrm{E}-07$ \\
ko01110 & Biosynthesis of secondary metabolites & $513(15.4 \%)$ & $333(16.49 \%)$ & $696(13.35 \%)$ & $2.20 \mathrm{E}-13$ & $2.01 \mathrm{E}-12$ & $8.29 \mathrm{E}-07$ \\
ko00941 & Flavonoid biosynthesis & $68(2.04 \%)$ & $49(2.43 \%)$ & $82(1.57 \%)$ & $2.08 \mathrm{E}-07$ & $1.10 \mathrm{E}-07$ & $1.74 \mathrm{E}-04$ \\
ko00945 & Stilbenoid, diarylheptanoid and gingerol biosynthesis & $62(1.86 \%)$ & $52(2.57 \%)$ & $75(1.44 \%)$ & $6.82 \mathrm{E}-07$ & $2.76 \mathrm{E}-10$ & $3.43 \mathrm{E}-04$ \\
ko04075 & Plant hormone signal transduction & $235(7.05 \%)$ & $145(7.18 \%)$ & $327(6.27 \%)$ & $6.98 \mathrm{E}-06$ & $3.30 \mathrm{E}-04$ & $1.14 \mathrm{E}-03$ \\
ko00073 & Cutin, suberine and wax biosynthesis & $48(1.44 \%)$ & $37(1.83 \%)$ & $39(0.75 \%)$ & $1.12 \mathrm{E}-12$ & $1.87 \mathrm{E}-12$ & $2.46 \mathrm{E}-03$ \\
\hline
\end{tabular}

signaling at stage $\mathrm{F}$ and activates this pathway via a low level of expression at stages $\mathrm{S}, \mathrm{T}$ and FO.

In total, 50 ARFs (Auxin response factors) were identified in our transcriptomic data using getorf [19] with minsize set to 150 and hmmsearch [20] with all parameters set to default according to descriptions in PlantfDB. After filtering those with low FPKM values, we obtained nine DEGs encoding ARFs (Additional file 9: Table S9); the FPKM value of CL8419.Contig2_All was significantly higher than that of the other eight DEGs (Additional file 9: Table S9). In F-vs-S paired comparisons, most of these DEGs were either upregulated or downregulated. The induction of auxin biosynthesis at stage $S$ via upregulation of $Y U C$ may activate or repress the expression of ARFs.

\section{Auxin quantification and localization}

A quantitative analysis of auxin levels at the four ovary development stages by HPLC-MS/MS revealed that levels were higher at stages $\mathrm{S}$ and $\mathrm{FO}$ than at the other two stages $(P<0.05)$ (Fig. 5$)$, indicating that auxin biosynthesis is activated after pollination at stage $S$. This result was consistent with high expression of $Y U C$ and PIN starting from stage $\mathrm{S}$.

An immunohistochemical analysis showed that auxin was uniformly distributed on the stigma and ovary primordium before pollination (Fig. 6a, g). After pollination, staining of the stigma increased compared to that at stage $\mathrm{S}$. The staining for auxin was especially intense at the bottom of the stigma where primordial ovary cells were located (Fig. 6b, h). Auxin was mainly located between cell walls (Fig. 6b). The rear part of the stigma, where the cavity for the pollen tube is located, remains viable for a long period after pollination [5]. After rudimentary ovary formation at stage $S$, the auxin staining in the pollen tube cavity of the stigma increased, and the auxin staining of the rudimentary ovary decreased (Fig. 6c, d and i). When the ovule began to form at stage $\mathrm{T}$, auxin staining was much intenser in ovules than in the parenchyma (Fig. 6e, j). During growth of the ovule at stage FO, auxin staining was much higher in the nucellus of the ovule than in the integument (Fig. 6f, $\mathrm{k}$ and l). Taken together, auxin is enriched at the growth center of pistillate inflorescences and young ovary after pollination.

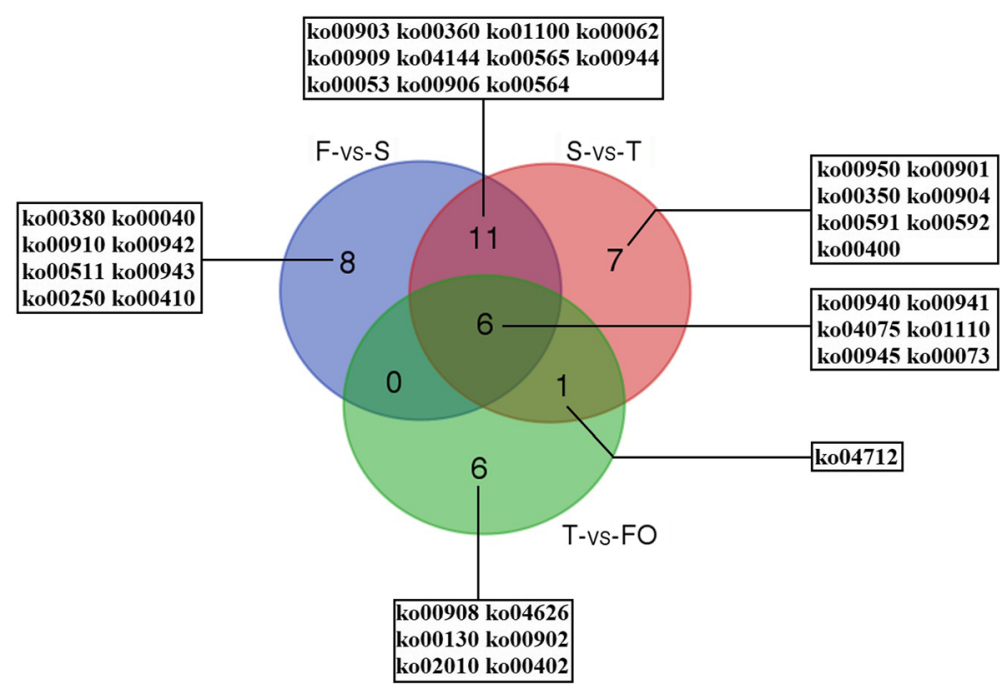

Fig. 4 Venn diagram showing the overlaps of significantly enriched pathways in F-vs-S, S-vs-T and T-vs-FO paired comparisons 


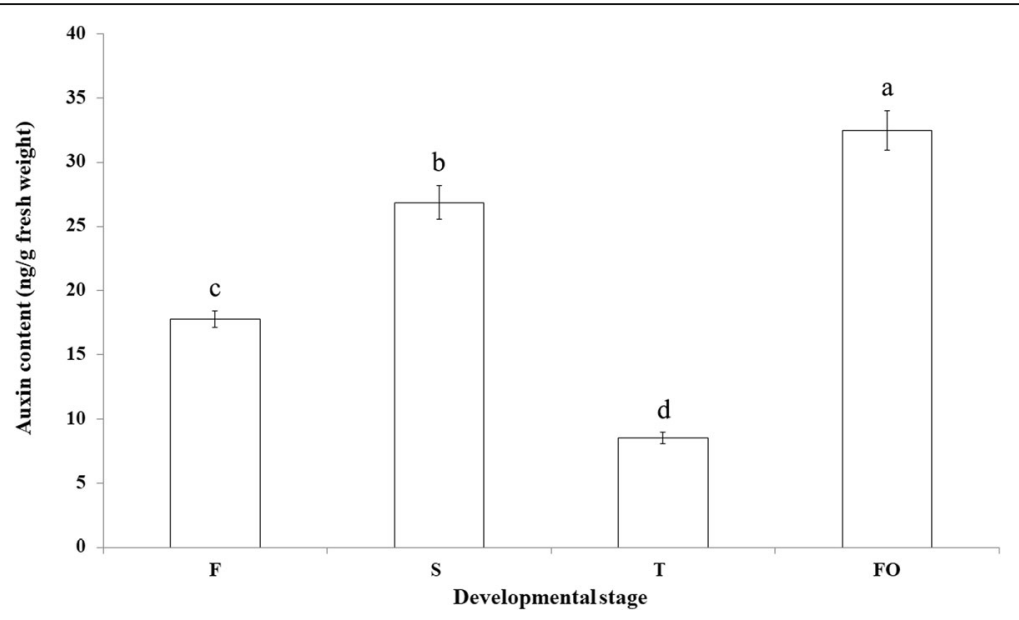

Fig. 5 Auxin content at different developmental stages. Different small letter indicate significant difference $(P<0.05)$ by LSD test $(n=3)$

DEGs encoding the regulator of flower development We identified a set of DEGs encoding quaternary proteins, including AGAMOUS (AG), SHATTERPPOOF (SHP), APETALA (AP)1, SEPALLATA (SEP)1, and SEEDSTICK (STK) (Additional file 11: Table S11). In F-vs-S paired comparisons, three DEGs encoding AG (CL5014.Contig1_ All, CL7712.Contig2_All, and Unigene17980_All) were downregulated 2.28, 2.05, and 1.99-fold, respectively; a DEG encoding SHP was downregulated 2.19-fold; two DEGs encoding SEP1 (Unigene19913_All and Unigene23003_All) were downregulated 1.35 and 3.63fold. Thus, the expression of these FQM (floral quartet model) components was relatively high at stage $\mathrm{F}$, and AP1 (Unigene28326_All) was highly expressed at all four developmental stages (Additional file 12: Table S12).

At stage $\mathrm{S}$, the FPKM values of AP1 (Unigene28326_All) and AG (Unigene17980_All) were 123.38 and 29.33, respectively (Additional file 12: Table S12). At stages $\mathrm{T}$ and FO, ovules in pistillate inflorescences begin to differentiate and grow. Three DEGs with high FPKM values including AP1 (Unigene28326_All), SHP (CL7712.Contig1_All), and $A G$ (Unigene17980_All) were upregulated (Additional file 11: Table S11). Thus, the differential expression of $A P 1, S H P, A G$, and SEP in the three paired comparisons and their relatively high FPKM values at specific developmental stages indicate that they are actively transcribed during ovary differentiation and growth.

Two DEGs encoding NGA (NGATHA) (CL1775.Contig9_All and Unigene14244_All) were identified in the F-vs-S paired comparisons (Additional file 11: Table S11); both DEGs were downregulated. Their maximum FPKM values occurred at stage $F$, and decreased thereafter and remained at a low level from stage $S$ to $F O$ (Additional file 10: Table S10).

The FPKM value of the DEG (Unigene23532_All) encoding KAN1 (Transcription repressor KAN1) was maximal at stage $\mathrm{F}$ and then decreased and remained at a low level from stages $\mathrm{S}$ to FO (Additional file 10: Table S10). This is similar to the pattern observed for $N G A$. The change in FPKM value of the DEG encoding PHBLIKE (Unigene26102_All) was similar to that of KAN1, and the value was much lower than that of KAN1 at the same developmental stage.

In total, five DEGs encoding FLOWERING LOCUS (FL) $C$ were identified in the three paired comparisons (Additional file 11: Table S11). Among these, CL5874.Contig1_ All had the highest FPKM value, and the FPKM values of all five DEGs decreased over the course of pistillate inflorescence development (Additional file 10: Table S10).

Only one DEG encoding FLOWERING LOCUS $(F)$ $T$ (CL10184.Contig4_All) was identified, and its FPKM value was $<2.0$ at the four developmental stages (Additional file 10: Table S10), indicating that it is expressed at a low level.

Forty-five DEGs encoding CONSTANS or CONSTANS-LIKE were identified; eight remained after filtering out those with low FPKM values. Most of these DEGs had their highest FPKM value at stage FO (Additional file 10: Table S10).

Three DEGs encoding SUPPRESSOR OF OVEREXPRESSION OF CONSTANS (SOC)1 (CL9612.Contig1_ All, CL9612.Contig2_All, and Unigene18364_All) were identified in S-vs-T paired comparisons; these DEGs were downregulated 2.70, 2.90, and 5.31-fold (Additional file 11: Table S11). These results indicate that SOC1 is more highly expressed at developmental stages $\mathrm{F}$ and $\mathrm{S}$ and regulates stigma growth and ovary primordium differentiation.

A DEG encoding LEAFY-LIKE PROTEIN (LFY) (Unigene3236_All) was downregulated 8.08-fold in the Svs-T paired comparisons (Additional file 11: Table S11), with FPKM values of 36.48, 27.16, 0.07, and 0.07 at stages 

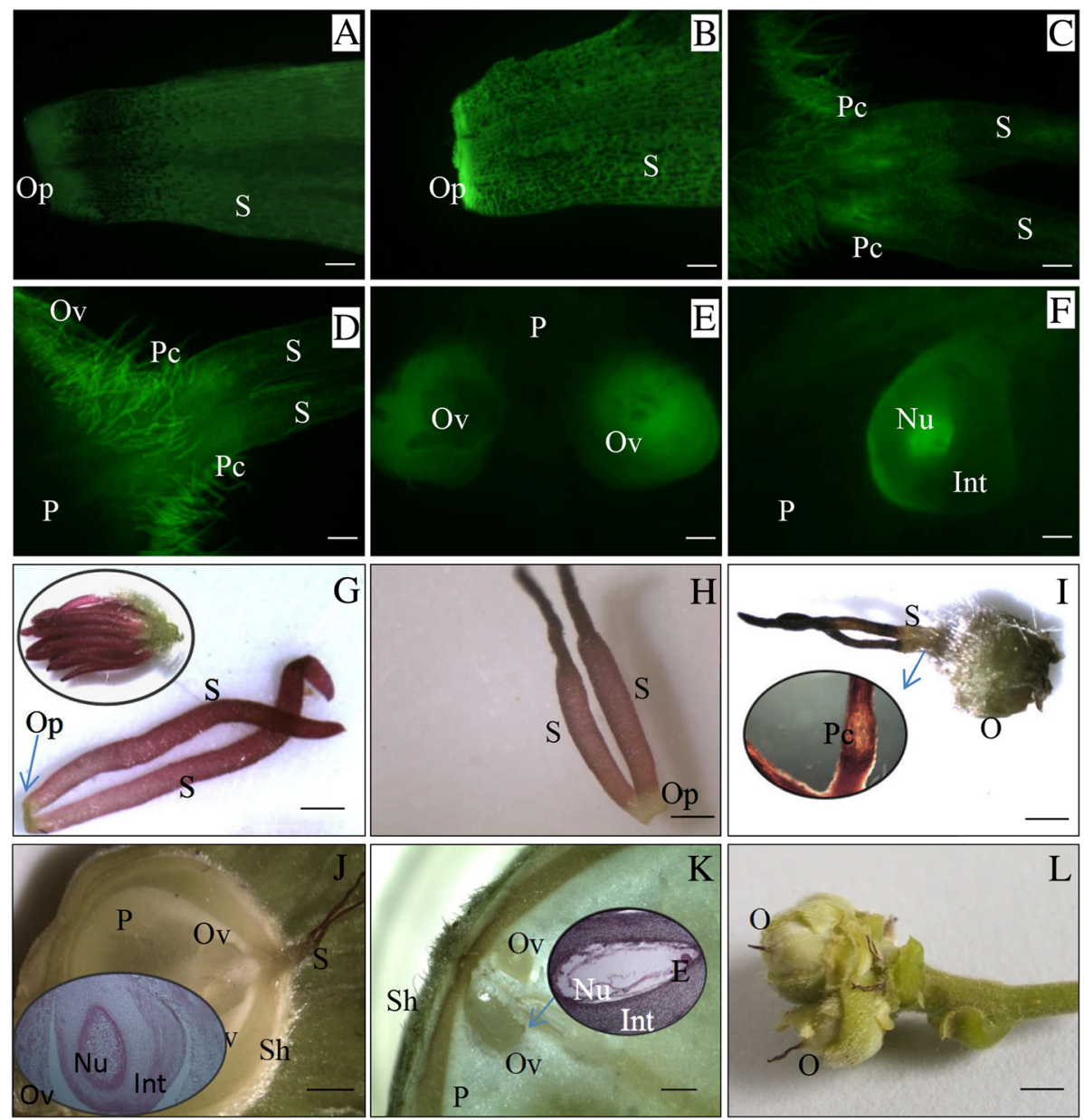

Fig. 6 Immunohistochemical analysis of auxin distribution at different developmental stages. a At stage F, auxin is approximately evenly distributed throughout the stigma and early ovary primordial; the patterns of staining in female inflorescences and single female flower with two stigmas are shown in (g). b Intenser auxin staining is observed in ovary primordia at stage S; stigmas with ovary primordia are shown in (h). c and $\mathbf{d}$ Intenser auxin staining are observed in the pollen tube cavity in stigmas after rudimentary ovary formation at stage S (May 10 and May 15); an early rudimentary ovary and its pollen tube cavity are shown in (i). e Auxin staining in ovules is much higher than in other tissues of early fruit at stage T; an ovary with two ovules is shown in (j). f Auxin content in the nucellus of ovules is much higher than in other tissues of early fruit at stage FO; an ovary with ovules, and the microstructure of ovule and fruit cluster are shown in (k) and (I). Key: S, stigma; Op, ovary primordial; PC, pollen tube cavity; O, ovary; Ov, ovule; P, parenchyma; Int, integument; Nu, nucellus; E, embryo; Sh, shell. Scale bars: $A, B=150 \mu \mathrm{m}, \mathrm{C}, \mathrm{D}, \mathrm{E}$ and $\mathrm{F}=300 \mu \mathrm{m} ; \mathrm{G}, \mathrm{H}=400 \mu \mathrm{m} ; \mathrm{I}=800 \mu \mathrm{m} ; \mathrm{J}$, $\mathrm{K}=1 \mathrm{~mm} ; \mathrm{L}=5 \mathrm{~mm}$

F, S, T, and FO, respectively (Additional file 10: Table S10); this pattern of variation in expression suggests a potential regulatory role at stages $\mathrm{F}$ and $\mathrm{S}$.

Two DEGs encoding GIGANTEA and 12 encoding SHORT VEGETATIVE PHASE (SVP) were observed in the three paired comparisons (Additional file 11: Table S11). GIGANTEA expression was lowest at stage FO, and its FPKM value was much higher than that of SVP (Additional file 10: Table S10), indicating low abundance of $S V P$.

\section{Validation of DEGs by qRT-PCR}

From the DEGs identified in the F-vs-S, S-vs-T, and Tvs-FO paired comparisons, 13 genes that may be involved in the regulation of ovary differentiation and development were selected for validation by qRT-PCR analysis: AP1, SHP, AG, SEP, FLC, CONSTANS, SOC1, GIGANTEA, LFY, YUC, AUX/IAA, ARF6, and ARF8 (Fig. 7). The expression patterns of these genes obtained in the qRT-PCR analysis were consistent with those from RNA sequencing.

\section{Discussion}

Auxin plays essential roles in organ patterning of pistillate inflorescences

Floral differentiation and development are complex biological processes that begin with flower bud induction and flower formation, and are regulated by 

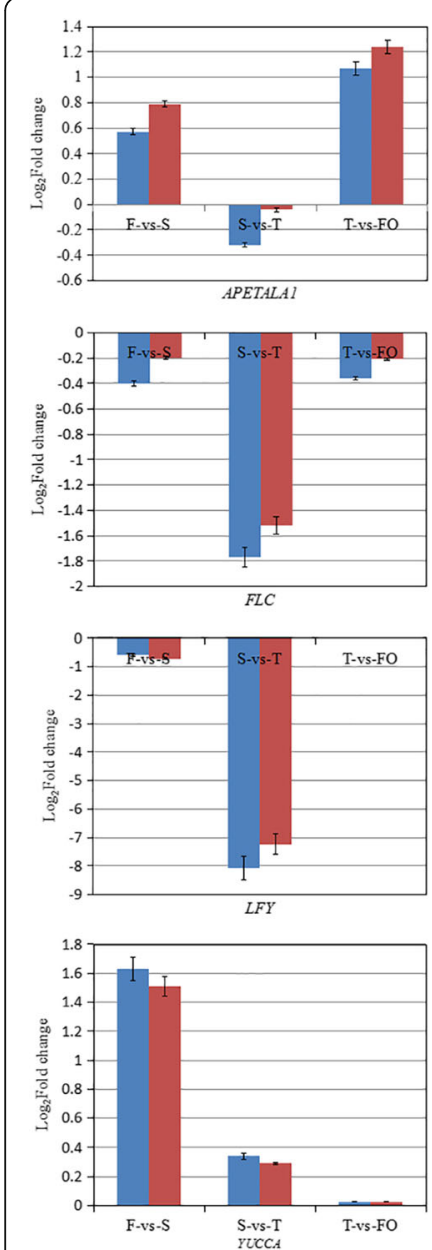
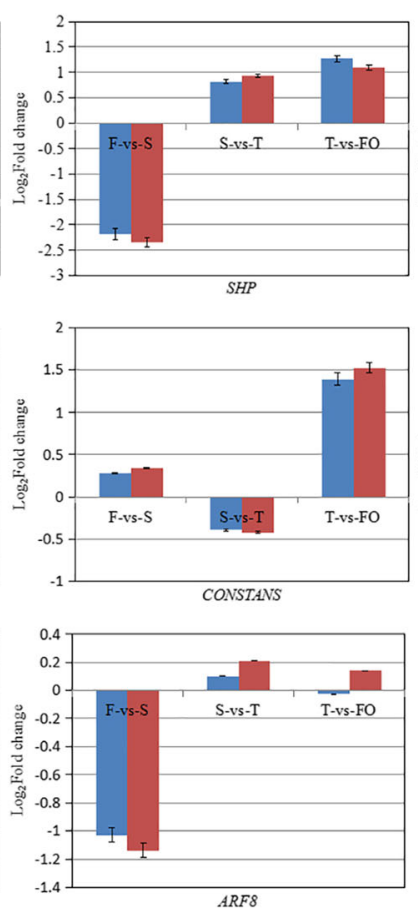

RNA-seq
QRT-PCR
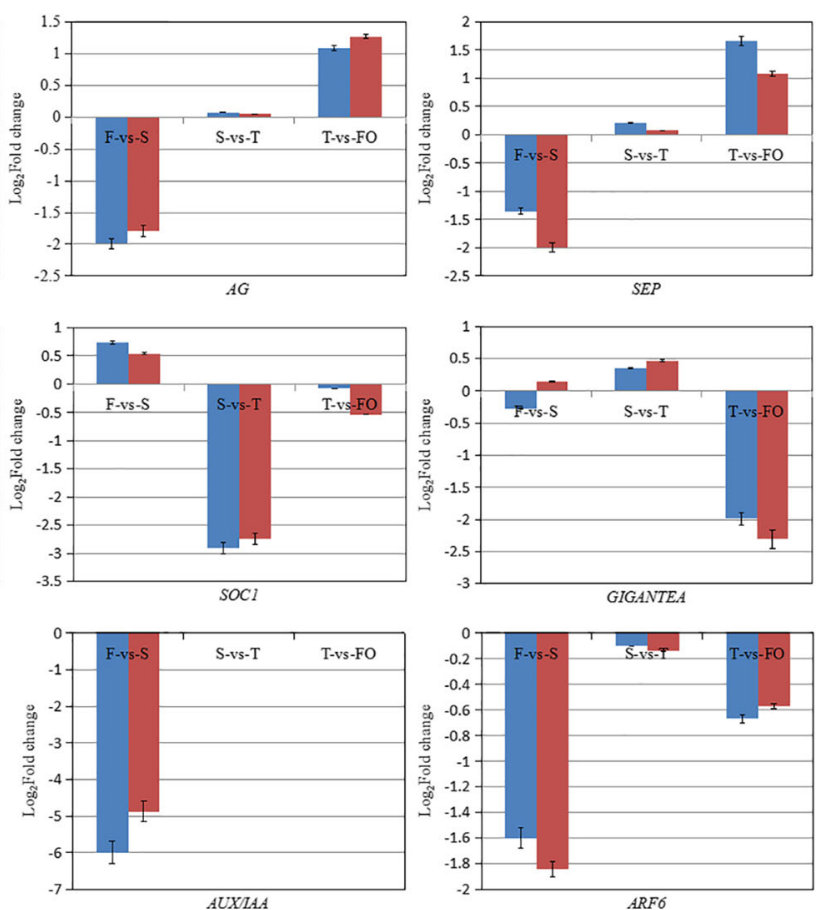

Fig. 7 Validation of DEGs by qRT-PCR analysis. Bars represent mean \pm standard deviation $(n=3)$

environmental factors (e.g., temperature, light quality, and water) [21, 22] and endogenous signals [23]. The phytohormone auxin is critical for plant morphogenesis; its local accumulation and distribution within plant organs provide spatial information for organ patterning [24]. In reproductive tissues, auxin metabolism, intercellular transport, and intracellular signaling coordinate the development of the stamen [24-26], gynoecia [27], ovule [28], and embryo [29].

Typical gynoecia consist of four parts, namely, the stigma, style, ovary, and gynophore; these structures are arranged along an apical-basal axis. High and low auxin concentrations in the apical and basal regions, respectively, create an auxin gradient that determines tissue specification and formation [2, 30]. Accordingly, changes in the auxin gradient, for example by treatment with the auxin transport inhibitor $\mathrm{N}$-1-naphthylphthalamic acid [31] or in mutants of auxin efflux (e.g., PIN1) [26] and auxin signaling (ARF5/MONOPTEROS and ARF3/ ETTIN) genes [32], result in aberrant gynoecium development. The IPA pathway is the major auxin biosynthetic pathway in plants and is essential for development of both flowers and fruits. YUC family proteins catalyze the rate-limiting conversion of IPA to IAA [33]. We found here that DEGs encoding $Y U C$ and the auxin efflux carrier gene PIN were upregulated in S-vs-T paired comparisons, and that auxin content was elevated at stage $\mathrm{S}$, implying active auxin biosynthesis and transport. An immunohistochemical analysis of auxin localization showed that auxin was enriched in primordial cells of the ovary. Thus, the auxin gradient in the ovary primordium is generated by the upregulation of $Y U C$ and PIN, which may be important for early ovary patterning and differentiation.

At anthesis in pistillate inflorescences, auxin was evenly distributed along the stigma. After pollination, ovary primordial cells at the bottom of the stigma accumulated auxin; this pattern of accumulation is similar to the higher auxin content in primordial leaf cells during asymmetry patterning in the gynoecium $[25,34]$. The auxin influx carrier protein AUX1 is critical for polarized transport between neighboring cells and establishment 
of concentration gradients across plant tissues [35]. During early differentiation of the ovary primordium (stage S), AUX1 downregulation and concomitant upregulation of PIN may result in the accumulation of auxin in primordial ovary cells and formation of an auxin gradient, with AUX/IAA, a negative regulator of auxin signaling, remaining low from stages $\mathrm{S}$ to $\mathrm{F}$. Thus, auxin signaling from stage $S$ to stage $F O$ may promote gynoecium asymmetry patterning and ovary primordium differentiation.

$A R F 6$ and ARF8 regulate the development of the gynoecium and stamen in immature flowers of Arabidopsis, including inflorescence stem elongation, anther dehiscence, gynoecium maturation, and flower bud opening [36, 37]. The ARF6-2/ARF8-3 double mutant exhibits sterility of male and female inflorescences and produces no seeds [36]; ectopic expression ARF6 or $A R F 8$ due to mutations in the microRNA miR167 causes growth arrest of ovule integuments [36]. Our transcriptome analysis revealed four DEGs encoding ARF6 and one encoding ARF8. All of these were downregulated in the F-vs-S paired comparisons, indicating their high expression at stage $\mathrm{F}$; their FPKM values remained at a relatively low level from stage $\mathrm{S}$ to stage FO. Thus, $A R F 6$ and $A R F 8$ likely play an important role in the regulation of stigma growth. However, their low levels during ovule differentiation and growth are not consistent with the sterility observed in the ARF6-2/ARF8-3 double mutant; additional studies are needed to determine whether ARF6 and $A R F 8$ are enriched in the ovule and modulate its growth.

\section{FQM components that regulate ovary and ovule differentiation and growth in hazelnut}

The FQM suggests that tetrameric complexes of floral homeotic proteins determine floral organ identity [38]. Carpel identity is determined by a complex of two SEP and two AG proteins, while ovule identity is determined by a complex of one SEP and one AG protein along with one each of the class D proteins, SHP and/or STK. Unlike most angiosperms, stamens are absent throughout the developmental cycle of the pistillate inflorescences of hazel; in particular, stigma growth and ovary and ovule growth and differentiation are initiated in a strict temporal sequence, providing a unique model for floral development. Based on their differential expression and FPKM values, AP1 (Unigene28326_All), SHP (CL7712.Contig1_All), AG (Unigene17980_All), and SEP (Unigene19913_All) were identified as the most important floral homeotic determinants of floral organ identity and are presumed to be associated with stigma identity. $A P 1$ and $A G$ may determine early ovary differentiation after pollination at stage $\mathrm{S}$, and along with SHP may regulate ovule differentiation and growth at stages $\mathrm{T}$ and FO. AP1 expression was significantly enhanced after pollination at stage $S$, implying regulation by auxin. We also found that ARF3 is a direct target of AP2 and partly mediates AP2 function in the determination of the floral meristem [39], although further investigation is required to clarify whether AP1 regulates ARF6 or ARF8 expression to promote crosstalk between the four homeotic proteins and the auxin signaling pathway.

Full-length $A G$ (CaMADS1) has been cloned from pollinated styles of hazel, and northern blot and RT-PCR analyses have confirmed its role in floral organogenesis [40]. A comparison of AG (Unigene17980_All) and CaMADS1 (GenBank: AF027376.1) sequences revealed 99\% similarity. Furthermore, constitutive expression of CaMADS1 in transgenic Arabidopsis resulted in the homeotic conversion of the first and second whorl organs [41]. Our results confirm the regulatory role of $A G$ not only in floral organogenesis but also in ovule development. In particular, FPKM values of AP1 during the four developmental stages were much higher than those of most DEGs encoding SHP, AG, SEP, and STK, highlighting the critical role of $A P 1$ in stigma growth, ovary differentiation, and ovule differentiation and growth. Whether AP1 is also involved in the regulation of floral differentiation remains to be determined.

\section{Important regulators of flower development contribute to ovary and ovule differentiation and growth after pollination in hazelnut}

$N G A$ genes interact with auxin signaling components at multiple levels throughout pistil development [42, 43]. The NGA family in Arabidopsis thaliana comprises four members that belong to the B3-type transcription factor superfamily. In the developing apical gynoecium of $N G A$ mutants, auxin synthesis is reduced via downregulation of $Y U C 8$, and the expression of genes involved in auxin transport and ARFs is altered throughout gynoecium development [43]. Here, we found that the maximum FPKM values of $N G A$ genes occurred at stage $\mathrm{F}$ in the stigma of female inflorescences; however, from stage $S$ to stage FO, FPKM values were low. These findings suggest that NGA genes regulate stigma growth.

PHB-LIKE proteins are important for apical meristem establishment and maintenance as well as adaxial organ identity [44], and are regulated by abaxial KAN proteins [45]. The antagonism between KAN and PHB-LIKE not only generates auxin gradients but also influences vascular development and establishment of organ asymmetry [32]. In the hazel transcriptome, $P H B$ was a DEG with low abundance; this adaxial factor may be overridden by $K A N 1$, which may determine the adaxial fate of the ovary primordium at stage $\mathrm{F}$ and provide a basis for stable ovary primordium partitioning.

$F L C$ is a MADS-box gene and strong suppressor of flowering. The expression of $F L C$ with low levels of 
DNA methylation is repressed by vernalization, resulting in early flowering [46]. In the vernalization and autonomous pathways, $F T$ expression is negatively regulated by $F L C$ [47]. Our results indicate that FLC expression decreased with pistillate inflorescence development, while the FT FPKM values remained at a low level throughout the four developmental stages. Vernalization is required before the anthesis of male and female inflorescences in hazel; inflorescences differentiate in the autumn and anthesis occurs in the following spring. The high level of FLC expression at stage $\mathrm{F}$ indicated the termination of vernalization. Additionally, as a strong suppressor of flowering, the low expression of $F L C$ from stages $S$ to FO implies the end of flowering suppression after pollination, which may facilitate subsequent development of pistillate inflorescences.

In order to complete their life cycle, higher plants must undergo a major developmental transition from vegetative growth to flowering. In many plants, flowering time is controlled by both environmental and endogenous signals. CONSTANS, which is related to GATA transcription factors, regulates the long-day pathway [48] and promotes flowering through transcriptional activation of $L F Y$, a flower meristem identity gene [49]. The highest FPKM values of CONSTANS were at stage FO, while $L F Y$ was highly expressed at stages $\mathrm{F}$ and $\mathrm{S}$. Thus, CONSTANS did not positively regulate $L F Y$, which is contrary to previous reports [49] and underscores the complexity of floral induction. The roles of CONSTANS and $L F Y$ in the regulation of ovule growth and of stigma and early ovary primordial differentiation merit further examination.

SOC1 is a MADS-box protein that is highly expressed in reproductive organs and promotes flowering in $A$. thaliana and Zea mays and regulates flowering by integrating multiple signals [50-52]. SOC1 expression levels were higher at developmental stages $\mathrm{F}$ and $\mathrm{S}$, suggesting that SOC1 promotes stigma growth and ovary primordium differentiation.

GIGANTEA induces flower differentiation in A. thaliana [53]. We found it to be highly expressed at stages F, $\mathrm{S}$, and $\mathrm{T}$, implying that it controls development of the stigma and differentiation of the ovary primordium and ovule at these developmental stages.

\section{Conclusions}

We identified a set of genes that may regulate stigma growth, primordial ovary formation, ovule differentiation and ovule growth in hazel. At stage F, four FQM components (AP1,SHP, $A G$, and $S E P)$ are highly expressed in pistillate inflorescences and which may determine floral identity and stigma growth; FLC and AUX/IAA suppress flowering, while SOC1, GIGANTEA, LFY, ARF6, ARF8, $N G A$, and KAN1 are highly expressed and act as positive inducers. Thus, the balance between positive and negative regulatory factors may determine stigma growth. Similarly, AP1, AG, YUC, PIN, CONSTANS, SOC1, GIGANTEA, LFY, ARF6, ARF8 and FLC may coordinate primordial ovary formation; AP1, SHP, AG, CONSTANS, GIGANTEA, YUC and PIN may determine ovule differentiation at stage $\mathrm{T}$, and $A P 1, S H P, A G, S E P, C O N$ STANS, YUC and PIN may regulate ovule growth.

\section{Additional files}

Additional file 1: Table S1. Primer sequences of selected unigenes in qRT-PCR. (XLSX $10 \mathrm{~kb})$

Additional file 2: Table S2. Categorization and abundance of reads. (XLSX $11 \mathrm{~kb}$ )

Additional file 3: Table S3. DEGs in F-vs-S paired comparisons. (XLSX 4773 kb)

Additional file 4: Table S4. DEGs in S-vs-T paired comparisons. (XLS $3850 \mathrm{~kb}$ )

Additional file 5: Table S5. DEGs in T-vs-FO paired comparisons. (XLS $3651 \mathrm{~kb}$ )

Additional file 6: Table S6. KEGG pathway enrichment analysis of DEGs identified in F-vs-S paired comparisons. (XLSX 23 kb)

Additional file 7: Table S7. KEGG pathway enrichment analysis of DEGs identified in S-vs-T paired comparisons. (XLSX $22 \mathrm{~kb}$ )

Additional file 8: Table S8. KEGG pathway enrichment analysis of DEGs identified in T-vs-FO paired comparisons. (XLSX 23 kb)

Additional file 9: Table S9. Selected DEGs in auxin biosynthesis, transport, and signal transduction pathway. (XLSX $12 \mathrm{~kb}$ )

Additional file 10: Table S10. FPKM values of all expressed transcripts. (XLS $7921 \mathrm{~kb}$ )

Additional file 11: Table S11. DEGs encoding regulators of flower development. (XLSX 12 kb)

Additional file 12: Table S12. FPKM values of DEGs encoding constituents of the floral quartet model (FQM). (XLSX $10 \mathrm{~kb})$

\section{Abbreviations}

AG: AGAMOUS; AP: APETALA1; ARF6: Auxin response factor 6; ARF8: Auxin response factor 8; AUX: Auxin influx transporter AUX; AUX/IAA: Auxin/ INDOLE-3-ACETIC ACID; FLC: FLOWERING LOCUS C; LFY: LEAFY; NGA: NGATHA; PIN: Auxin efflux carrier family protein PIN; SEP: SEPALLATA 1; SHP: SHATTERPPOOF; SOC1: SUPPRESSOR OF OVEREXPRESSION OF CONSTANS1

\section{Acknowledgements}

We thank Dr. Ji for critical reading and editing the manuscript.

\section{Funding}

This work was financially supported by the National Natural Science Foundation of China (no. 31770723; 31670681; 31370683). The funders had no role in study design, data collection and analysis, decision to publish, or preparation of the manuscript.

\section{Availability of data and materials}

The raw sequence data have been deposited in the National Center for Biotechnology Information (NCBI) database (https://www.ncbi.nlm.nih.gov/sra/ ?term=SRS1980648). All other relevant supplementary data is provided within this manuscript as Additional files 1, 2, 3, 4, 5, 6, 7, 8, 9, 10, 11, 12: Tables S1-S12.

\section{Authors' contributions}

$J \mathrm{~L}$ and $Y C$ contributed to the design of the experiments, collection and/or assembly of data, data analysis and interpretation, and manuscript writing. YZ and YC carried out experiments. YZ, CL and PA prepared samples, 
collected and/or assembled data, and analyzed and interpreted data. All authors read and approved the final manuscript.

\section{Ethics approval and consent to participate} Not applicable.

\section{Competing interests}

The authors declare that they have no competing interests.

\section{Publisher's Note}

Springer Nature remains neutral with regard to jurisdictional claims in published maps and institutional affiliations.

\section{Author details}

${ }^{1}$ Jilin Provincial Key Laboratory of Plant Resource Science and Green Production, Jilin Normal University, Siping 136000, Jilin Province, China. ${ }^{2}$ College of Bioscience \& Bioengineering, Hebei University of Science and Technology, Shijiazhuang 050080, Hebei Province, China.

\section{Received: 10 October 2017 Accepted: 26 April 2018} Published online: 09 May 2018

\section{References}

1. Fideghelli C, Salvador FRD, Varvaro L, Franco S. World hazelnut situation and perspectives. Acta Horticul. 2009;845:39-52.

2. Larsson E, Franks RG, Sundberg E. Auxin and the Arabidopsis thaliana gynoecium. J Exp Bot. 2013;64:2619.

3. Smyth DR, Bowman JL, Meyerowitz EM. Early flower development in Arabidopsis Plant Cell. 1990;2:755-67.

4. Ciampolini F, Cresti M. The structure and cytochemistry of the stigma-style complex of Corylusavellana L. 'Tonda gentile delleLanghe' (Corylaceae). Ann Bot. 1998;81:513-8.

5. Liu J, Zhang H, Cheng Y, Kafkas S, Güney M. Pistillate flower development and pollen tube growth mode during the delayed fertilization stage in Corylus heterophylla Fisch. Plant Reprod. 2014;27:145-52.

6. Liu J, Zhang H, Cheng Y, Wang J, Zhao Y, Geng W. Comparison of ultrastructure, pollen tube growth pattern and starch content in developing and abortive ovaries during the progamic phase in hazel. Front Plant Sci. 2014:5:528.

7. Cheng Y, Zhao Y, Liu J, Yang B, Ming Y. Comparison of phytohormone biosynthesis and signal transduction pathways in developing and abortive hazelnut ovules. Plant Growth Regul. 2017;811:147-57.

8. Liu J, Cheng Y, Yan K, Liu Q, Wang Z. The relationship between reproductive growth and blank fruit formation in Corylus heterophylla Fisch. Sci Hortic. 2012;136:128-34.

9. Liu J, Ming Y, Cheng Y, Zhang Y, Xing J, Sun Y. Comparative transcriptome analysis reveal candidate genes potentially involved in regulation of primocane apex rooting in raspberry (Rubus spp). Front Plant Sci. 2017:8:1036.

10. Grabherr MG, Haas BJ, Yassour M, Levin JZ, Thompson DA, Amit I, Adiconis X, Fan L, Raychowdhury R, Zeng Q, Chen Z, Mauceli E, Hacohen N, Gnirke A, Rhind N, di Palma F, Birren BW, Nusbaum C, Lindblad-Toh K, Friedman N, Regev A. Full-length transcriptome assembly from RNA-Seq data without a reference genome. Nat Biotechnol. 2011;29:644-52.

11. Pertea G, Huang X, Liang F, Antonescu V, Sultana R, Karamycheva S, Lee $Y$, White J, Wheung F, Parvizi B, Tsai J, Quackenbush J. Tigr gene indices clustering tools (tgicl): a software system for fast clustering of large EST datasets. Bioinformatics. 2003;19:651-2.

12. Altschul SF, Gish W, Miller W, Myers EW, Lipman DJ. Basic local alignment search tool. J Mol Bio. 1990:215:403-10.

13. Conesa A, Götz S, Garcíagómez JM, Terol J, Talón M, Robles M. Blast2go: a universal tool for annotation visualization and analysis in functional genomics research. Bioinformatics. 2005;21:3674-6.

14. Quevillon E, Silventoinen V, Pillai S, Harte N, Mulder N, Apweiler R, Lopez R. Interproscan: protein domains identifier. Nucleic Acids Res. 2005;33:116-20.

15. Langmead B, Salzberg SL. Fast gapped-read alignment with bowtie 2 . Nat Methods. 2012;9:357-9.

16. Li B, Dewey CN. RSEM: accurate transcript quantification from RNA-Seq data with or without a reference genome. BMC Bioinformatics. 2011;12:323.

17. Leng N, Dawson JA, Thomson JA, Ruotti V, Rissman Al, Smits BM, Haag JD, Gould MN, Stewart RM, Kendziorski C. EBSeq: an empirical Bayes hierarchical model for inference in RNA-seq experiments. Bioinformatics. 2013;29:1035-43.
18. You C, Zhu H, Xu B, Huang WX, Wang SH, Ding YF, Liu ZH, Li GH, Chen L, Ding CQ, Tang S. Effect of removing superior spikelets on grain filling of inferior spikelets in rice. Front Plant Sci. 2016;7:1161.

19. Rice P, Longden I, Bleasby A. EMBOSS: the European molecular biology open software suite. Trends Genet. 2000;16:276.

20. Mistry J, Finn RD, Eddy SR, Bateman A, Punta M. Challenges in homology search: hmmer3 and convergent evolution of coiled-coil regions. Nucleic Acids Res. 2013;41:e121.

21. Li JX, Hou XJ, Zhu J, Zhou JJ, Huang HB, Yue JQ, Gao JY, Du YX, Hu CX, Hu CG, Zhang JZ. Identification of genes associated with lemon floral transition and flower development during floral inductive water deficits: a hypothetical model. Front Plant Sci. 2017;8:1013.

22. Christiaens A, Pauwels E, Gobin B, Labeke MCV. Flower differentiation of azalea depends on genotype and not on the use of plant growth regulators. Plant Growth Regul. 2015;75:245-52.

23. Sladký $Z$. The role of growth regulators in the differentiation of flowers and inflorescences. Biol Plantarum. 1986:28:31.

24. Benkova E, Michniewicz M, Sauer M, Teichmann T, Seifertova D, Jurgens G, Friml J. Local efflux-dependent auxin gradients as a common module for plant organ formation. Cell. 2003;115:591-602.

25. Ding Z, Wang B, Moreno I, Dupláková N, Simon S, Carraro N, Reemmer J, Pěnčík $A$, Chen X, Tejos R, Skůpa P, Pollmann S, Mravec J, Petrášek J, Zažímalová E, Honys D, Rolčík J, Murphy A, Orellana A, Geisler M, Friml J. ER-localized auxin transporter PIN8 regulates auxin homeostasis and male gametophyte development in Arabidopsis. Nat Commun. 2012;3:941.

26. Cecchetti V, Brunetti P, Napoli N, Fattorini L, Altamura MM, Costantino PP, Cardarelli M. The ABCB1 and ABCB19 auxin transporters have synergistic effects on early and late Arabidopsis anther development. J Integr Plant Biol. 2015;57:1089-98.

27. Moubayidin L, Ostergaard L. Dynamic control of auxin distribution imposes a bilateral-to-radial symmetry switch during gynoecium development. Curr Biol. 2014:24:2743-8.

28. Galbiati F, Sihna Roy D, Simonini S, Cucinotta M, Ceccato L, Cuesta C, Simaskova M, Benkova E, Kamiuchi Y, Aida M, Weijers D, Simon R, Masiero S, Colombo L. An integrative model of the control of ovule primordia formation. Plant J. 2013;76:446-55.

29. Robert HS, Grones P, Stepanova AN, Robles LM, Lokerse AS, Alonso JM, Weijers D, Friml J. Local auxin sources orient the apical-basal axis in Arabidopsis embryos. Curr Biol. 2013;23:2506-12.

30. Nemhauser JL, Feldman LJ, Zambryski PC. Auxin and ETTIN in Arabidopsis gynoecium morphogenesis. Development. 2000;127:3877-88.

31. Larsson E, Roberts CJ, Claes AR, Franks RG, Sundberg E. Polar auxin transport is essential for medial versus lateral tissue specification and vascular-mediated valve outgrowth in Arabidopsis gynoecia. Plant Physiol. 2014;166:1998.

32. Pekker I, Alvarez JP, Eshed Y. Auxin response factors mediate Arabidopsis organ asymmetry via modulation of KANADI activity. Plant Cell. 2005;17: 2899-910.

33. Mashiguchi K, Tanaka K, Sakai T, Sugawara S, Kawaide H, Natsume M, Hanada A, Yaeno T, Shirasu K, Yao H, McSteen P, Zhao Y, Hayashi K, Kamiya $\mathrm{Y}$, Kasahara $\mathrm{H}$. The main auxin biosynthesis pathway in Arabidopsis. Proc Natl Acad Sci U S A. 2011;108:18512-7.

34. Reinhardt D, Pesce ER, Stieger P, Mandel T, Baltensperger K, Bennett M, Traas J, Friml J, Kuhlemeier C. Regulation of phyllotaxis by polar auxin transport. Nature. 2003;426:255-60.

35. Yang Y, Hammes UZ, Taylor CG, Schachtman DP, Nielsen E. High-affinity auxin transport by the AUX1 influx carrier protein. Curr Biol. 2006;16:1123-7.

36. Nagpal P, Ellis CM, Weber H, Ploense SE, Barkawi LS, Guilfoyle TJ, Hagen G, Alons JM, Cohen JD, Farmer EE, Ecker JR, Reed JW. Auxin response factors arf6 and arf8 promote jasmonic acid production and flower maturation. Development. 2005;132:4107-18.

37. Wu MF, Tian Q, Reed JW. Arabidopsis microRNA167 controls patterns of ARF6 and ARF8 expression and regulates both female and male reproduction. Development. 2006;133:4211-8.

38. Theißen G, Melzer R, Rümpler F. MADS-domain transcription factors and the floral quartet model of flower development: linking plant development and evolution. Development. 2016;143:3259.

39. Liu X, Dinh TT, Li D, Shi B, Li Y, Cao X, Guo L, Pan Y, Jiao Y, Chen X. Auxin response factor 3 integrates the functions of AGAMOUS and APETALA2 in floral meristem determinacy. Plant J. 2014;80:629.

40. Rigola D, Pè ME, Fabrizio C, Mè G, Sarigorla M. CaMADS1, a MADS box gene expressed in the carpel of hazelnut. Plant Mol Biol. 1998;38:1147-60. 
41. Rigola D, Pè ME, Mizzi L, Ciampolini F, Sari-Gorla M. CaMADS1, an AGAMOUS homologue from hazelnut, produces floral homeotic conversion when expressed in Arabidopsis. Sex Plant Reprod. 2001;13:185-91.

42. Alvarez JP, Goldshmidt A, Efroni I, Bowman JL, Eshed Y. The NGATHA distal organ development genes are essential for style specification in Arabidopsis. Plant Cell. 2009;21:1373-93.

43. Irene MF, Sofía S, Naciele M, Vicente B, Patricia B, Marisa NG, Antonio CO, Lucia C, Cristina F. The effect of NGATHA altered activity on auxin signaling pathways within the Arabidopsis gynoecium. Front Plant Sci. 2014;5:210.

44. Prigge MJ, Otsuga D, Alonso JM, Ecker JR, Drews GN, Clark SE. Class II homeodomain-leucine zipper gene family members have overlapping antagonistic and distinct roles in Arabidopsis development. Plant Cell. 2005;17:61-76.

45. Mallory AC, Reinhart BJ, Jones-Rhoades MW, Tang G, Zamore PD, Barton MK, Bartel DP. MicroRNA control of PHABULOSA in leaf development: importance of pairing to the microRNA 5' region. EMBO J. 2004;23:3356-64.

46. Jean FE, Kovac KA, Jaligot E, Sheldon CC, James PW, Dennis ES. The downregulation of FLOWERING LOCUS C (FLC) expression in plants with low levels of DNA methylation and by vernalization occurs by distinct mechanisms. Plant J. 2005:44:420-32.

47. Hepworth SR, Valverde F, Ravenscroft D, Mouradov A, Coupland G. Antagonistic regulation of flowering-time gene SOC1 by CONSTANS and FLC via separate promoter motifs. EMBO J. 2002;21:4327.

48. Valverde F. CONSTANS and the evolutionary origin of photoperiodic timing of flowering. J Exp Bot. 2011;62:2453-63.

49. Blázquez MA. Illuminating flowers: CONSTANS induces LEAFY expression BioEssays. 1997;19:277.

50. Ding L, Wang Y, Yu H. Overexpression of DOSOC1, an ortholog of Arabidopsis SOC1, promotes flowering in the orchid Dendrobium Chao Parya smile. Plant Cell Physiol. 2013;54:595-608.

51. Immink R, Pose D, Ferrario S, Ott F, Kaufmann K, Valentim FL, Folter SD, Wal FVD, Dijk ADJV, Schmid M, Angenent GC. Characterization of SOC1's central role in flowering by the identification of its up- and downstream regulators. Plant Physiol. 2012;160:433-49.

52. Zhao S, Luo Y, Zhang Z, Xu M, Wang W, Zhao Y, Zhang L, Fan Y, Wang L. ZmSOC1, an MADS-box transcription factor from Zea mays promotes flowering in Arabidopsis. Int J Mol Sci. 2014;15:19987-20003.

53. Sawa M, Kay SA. GIGANTEA directly activates Flowering Locus $T$ in Arabidopsis thaliana Proc Natl Acad Sci U S A. 2011:108:11698-703.

\section{Ready to submit your research? Choose BMC and benefit from:}

- fast, convenient online submission

- thorough peer review by experienced researchers in your field

- rapid publication on acceptance

- support for research data, including large and complex data types

- gold Open Access which fosters wider collaboration and increased citations - maximum visibility for your research: over $100 \mathrm{M}$ website views per year

At BMC, research is always in progress.

Learn more biomedcentral.com/submissions 